\title{
Introdução: Festivais de cinema e os seus contextos socioculturais
}

\author{
Aida Vallejo \\ Universidade do País Basco UPV/EHU \\ aida.vallejo@ehu.eus \\ https://orcid.org/0000-0002-2433-0675 \\ Tânia Leão \\ Universidade do Porto - Instituto de Sociologia \\ tsilva@letras.up.pt \\ https://orcid.org/0000-0003-1670-075X
}

Quando escrevemos a chamada para artigos para este dossier especial da Aniki, em fevereiro de 2020, nada nos fazia prever que, um ano depois, todo o ecossistema dos festivais estaria paralisado devido a uma pandemia global. Seria, justamente, o foco temático que optámos por privilegiar - os contextos socioculturais em torno dos festivais cinematográficos - o mais afetado nesta época da denominada 'distância social'. Face à crise que se instalou, os diversos países apresentaram respostas governamentais distintas, aplicadas em fases, também elas, diferentes. As próprias organizações dos festivais foram testando soluções heterogéneas: desde o cancelamento inicial dos eventos, à sua recalendarização, à celebração presencial com medidas especiais adaptada aos protocolos de segurança (como a redução da taxa de ocupação das salas, o uso de máscaras, o estabelecimento de distâncias mínimas entre as pessoas, etc.), até à decisão de oferecer uma programação exclusivamente online, em regime híbrido (online e presencial), ou apenas presencial.

Tais mudanças levaram a uma reflexão, por parte da indústria e das estruturas que organizam os festivais, sobre as suas estratégias e modos de funcionamento. Nesta reflexão, a dimensão eminentemente social dos festivais ganhou especial relevância. Tal como referiu Marijke de Valck na sua crónica de urgência, escrita em plena primeira vaga de contágios (onde a autora analisava os encontros online organizados por festivais, procurando identificar desafios e soluções possíveis para a crise): 
(...) as tentativas, criativas, de mimetizar a experiência do festival online, revelaram-se mais complicadas do que se pensara. As conversas virtuais, os Q\&A's, as festas e os mercados diferem dos seus congéneres físicos, na atmosfera e nos recursos envolvidos. Quando o entusiasmo inicial da experimentação online diminuiu, e se instalou a fadiga do tempo passado em frente ao ecrã, verificou-se que os festivais virtuais são, simplesmente, menos festivos e, portanto, menos eficazes para alcançar alguns dos seus objetivos. (De Valck 2020, nossa tradução)

É por isso que, neste momento mais do que nunca, é imprescindível estudar a importância e a influência das relações sociais que ocorrem no contexto dos festivais, no que toca às culturas cinematográficas que estes geram, modelam ou questionam.

Os trabalhos de investigação apresentados neste número procuram responder a esse objetivo, analisando as diferentes formas a partir das quais a negociação social e a criação de discursos em torno dos festivais contribuíram para criar determinadas culturas cinematográficas. E fazem-no olhando para contextos como o Chile, Brasil, Senegal, Galiza (Espanha) e Portugal. Além disso, esta publicação apresenta-se como um trabalho pioneiro no âmbito lusófono, dando conta do interesse incipiente que os/as pesquisadores/as de Portugal e do Brasil têm revelado por esta área. Por esse motivo, este texto introdutório não será uma mera apresentação dos trabalhos coletados, mas, também, um texto de balanço, que visa oferecer uma revisão do estado da arte sobre a temática em apreço. Em primeiro lugar, traçaremos uma panorâmica geral do desenvolvimento e evolução da subdisciplina, relativamente jovem, dos Film Festival Studies. Num segundo momento, apresentaremos uma análise crítica dos estudos sociológicos e antropológicos que têm os festivais de cinema como objeto de estudo.

\section{Os Film Festival Studies}

O campo de estudo dos festivais de cinema tem tido, desde o seu início, um caráter fortemente interdisciplinar. Embora as autoras de referência tenham trabalhado em torno dos estudos de cinema (note-se que, neste caso, as principais referências são mulheres, uma peculiaridade no seio do mundo académico), muitas das pesquisas sobre festivais cinematográficos são trabalhos esporádicos oriundos de diversas disciplinas como a história, o marketing, a economia da cultura, o turismo, os estudos de género, a antropologia ou a sociologia, entre outras. Certamente, tal dever-se-á ao caráter plurifacetado do próprio objeto de estudo: os 
festivais, que, como se sabe, atuam em diferentes níveis e atendem a diversas agendas em simultâneo (muitas vezes, opostas). De facto, o livro pioneiro assinado por de Valck (2007) converteu-se numa referência fundamental para a disciplina, devido, justamente, ao olhar múltiplo que a autora faz incidir no fenómeno festival. Na análise que faz dos certames que se realizam no âmbito europeu, a autora dedica um estudo de caso a cada uma dessas dimensões: Berlim e a política internacional, Cannes e o mercado audiovisual, Veneza e a cobertura mediática, e Roterdão e a criação de públicos. Nesse trabalho, de Valck também propôs uma periodização histórica, aplicável entre os anos de 1930 e os inícios de 2000, que tem servido de referência a uma parte significativa de pesquisas subsequentes (ex. Wong 2011; Vallejo 2014) que têm pugnado pela ampliação do estudo dos festivais a outras partes do globo, com destaque para as periferias do circuito dos festivais (espaços culturais não hegemónicos).

Com efeito, uma das linhas de investigação que mais interessou às pesquisas sobre festivais de cinema, logo nos seus primórdios, foi a dos 'cinemas periféricos'. Dada a importância do circuito internacional de festivais para a circulação e legitimação, também internacional, de cineastas e movimentos cinematográficos (Vallejo 2020b), especialistas em cinematografias de diferentes regiões geopolíticas não-hegemónicas encontraram, nos festivais, um espaço fundamental para compreender (e aceder a) os seus objetos de estudos. É o caso de Dina Iordanova, para a cinematografia da Europa de Leste e, mais recentemente, para o cinema asiático (Iordanova e Cheung 2011; Iordanova 2017); de Lindiwe Dovey para o cinema africano (2015); e de Tamara Falicov $(2010,2017)$ ou Minerva Campos $(2012,2020)$, para o cinema latinoamericano. Os últimos, têm demonstrado especial interesse nos sistemas de coprodução facilitados pelos festivais. Mais recentemente, foi a análise das identidades no cinema (que já contava com uma longa história no campo dos estudos cinematográficos) que desembocou nos estudos de festivais. Simultaneamente, verificou-se uma proliferação de eventos especializados. Neste ponto, interessa destacar o extenso número de publicações dedicadas aos festivais LGBT+ (Loist e Zielinski 2012; Loist 2013; Richards 2016; Rhyne 2018; Damiens 2020a). Há ainda a salientar a "Film Festival Research Network" (co-fundada por Loist e de Valck), que oferece, no seu site, uma compilação bibliográfica permanentemente atualizada e em aberto dos estudos de festivais de cinema e que se tem revelado um ponto de encontro e um catalisador fundamental para a pesquisa nesta área. Por fim, a última década, viu a publicação de vários trabalhos que atualizaram a bibliografia 
de referência, introduzindo contributos essenciais. Foi o caso dos livros da extinta série "Film Festival Yearbook", editada por Iordanova entre 2009 e 2014, ou da sua sucessora, a série "Framing Film Festivals", da Palgrave MacMillan, coeditada por de Valck e Falicov desde 2015. Da mesma forma, as antologias de Iordanova (2013) e de de Valck, Kredell e Loist (2016) tornaram-se manuais de referência para esta área de estudos: o primeiro como uma coletânea de textos 'clássicos', e o segundo como compilação das principais linhas de investigação e enquadramentos teórico-metodológicos dos Film Festival Studies.

Na primeira fase de desenvolvimento dos estudos dos festivais de cinema, identificamos duas tendências principais. Em primeiro lugar, existiu uma vontade de discernir e estabelecer as bases teóricas que permitissem enquadrar todos os temas e aspectos passíveis de serem analisados no âmbito dos festivais. A abertura a novas disciplinas e metodologias tem sido, por isso mesmo, uma constante. Em segundo lugar, percebe-se ter havido uma ambição cartográfica. Ou seja, pretendeu-se realizar um inventário geolocalizado dos festivais, num momento em que estes se multiplicavam exponencialmente (a par, aliás, do desenvolvimento da própria disciplina). Tal propósito levou à inclusão, em várias publicações, de incontáveis tabelas e listas de eventos. Mas também de listagens de atividades relacionadas com festivais, em particular entre as publicações dedicadas a festivais especializados, como os festivais de direitos humanos (Iordanova e Torchin 2012), cinema etnográfico (Peirano 2017a) ou documentário (Vallejo 2020a); ou entre aqueles que ocorrem em certos países ou regiões geopolíticas, incluindo contextos nacionais/ estatais como França (Taillibert 2009) ou Espanha (Jurado 2003). Os livros das séries "Film Festival Yearbook" e "Framing Film Festivals" têm sido exemplares, desse ponto de vista. Além das publicações académicas, há ainda a assinalar diversos relatórios ou sites de pesquisa que têm contribuído para mapear os festivais em contextos nacionais: destacamos os trabalhos de Leal e Mattos sobre o Brasil (Mattos e Leal 2009; Leal e Mattos 2011) e de Peirano sobre o Chile (Peirano e González 2018). ${ }^{1}$

Como salientámos anteriormente, o interesse em cinematografias periféricas esteve, ele próprio, na origem dos estudos de festivais. Muitas dessas pesquisas foram levadas a cabo no âmbito da academia anglófona,

${ }^{1}$ Ver o site interativo sobre festivais chilenos: http://www.festivalesdecine.cl/ 
sobretudo por pesquisadores especializados em 'cinema de outras partes do mundo' (o que se convencionou chamar de world cinema ou, mais recentemente, cinema of the global south). Nos últimos anos, há a assinalar o aumento do interesse de revistas e jornais académicos, de diferentes áreas, pela publicação de números e dossiers especiais sobre esta temática. Tal tem contribuído para difundir o estudo dos festivais em novas áreas geográficas e lingüísticas - como fazemos, neste dossier, para o âmbito lusófono. Realçamos vários números especiais sobre festivais de cinema publicados em 2014, como o da revista Secuencias, pioneira na língua castelhana, editado por Aida Vallejo; o da Iluminace, em checo, editado por Jindřiška Bláhová; ou o da Cinergie, centrada no contexto italiano (em inglês, mas com trabalhos de pesquisadores/as italianos/as) e editado por Monica Acciari e Roy Menarini. Algumas publicações mais recentes incluem os números especiais dedicados aos festivais de cinema da América Latina, na revista argentina Cine Documental, editado por María Paz Peirano e Mariana Amieva, em 2018, e na revista chilena Comunicación y Medios, editado por Peirano e Vallejo, em 2020.

É possível perceber, através do itinerário que fomos traçando, que os festivais de cinema apresentam-se também como espaços fundamentais para se refletir sobre as relações de poder - não só no campo cinematográfico, mas também no académico. Uma vez que o cânone cinematográfico do cinema mundial foi moldado pela seleção e reconhecimento de determinados filmes e cineastas em festivais de grande repercussão internacional (como Cannes ou Veneza), consideramos necessário repensar o papel destes e de outros festivais no desenvolvimento e modelação de certas culturas cinematográficas. A melhor forma de o fazer é oferecendo "histórias geolocalizadas" de cinema que têm como referência festivais específicos (Vallejo 2020b). Fazendo-o, torna-se possível obter um conhecimento mais aprofundado dos poderes que, ao operarem dentro e fora dos contextos nacionais, influenciam-se mutuamente e concorrem para construir uma dada história nacional do cinema $(2020 b, 4)$. Ora, os artigos deste dossier temático, ao descreverem os processos socioculturais através dos quais determinados contextos cinematográficos se modelam e transformam a partir dos festivais, respondem precisamente a esta proposta. 


\section{O estudo socioantropológico dos festivais}

Entre os estudos que se dedicam à análise da dimensão sociocultural dos festivais, podemos encontrar diferentes linhas de investigação. A primeira dessas linhas, de natureza mais teórica e metodológica, propõe e reflete sobre conceitos e ferramentas para o estudo do festival de cinema como espaço social. A segunda, inclui estudos de caso que analisam as práticas de diversos agentes sociais que interagem no contexto dos festivais: trabalhadores do festival, profissionais que circulam pela 'rede' e públicos.

As obras de sociólogos como Bruno Latour (com a sua teoria do Ator-Rede), Pierre Bordieu (com a sua teoria dos campos sociais e proposta de análise dos processos de criação de valor simbólico) e Howard S. Becker (com a sua teoria dos 'Mundos da Arte' colaborativos, informais e flexíveis) têm sido amplamente utilizadas como referenciais teóricos no estudo dos festivais, servindo para situar a disciplina dentro do quadro mais amplo das ciências humanas e sociais. No campo dos estudos cinematográficos, a obra de Harbord (2002) oferece uma aproximação filosófica aos espaços de exibição e, mais concretamente, aos festivais de cinema - seguindo a corrente que tem deslocado o interesse da produção científica para as esferas da exibição e receção, amplamente desenvolvida pela new cinema history (ver Biltereyst, Maltby e Meers 2019). Por outro lado, as pesquisas dedicadas à cinefilia têm proporcionado reflexões sobre o comportamento das audiências, reivindicando novas cinefilias, no plural (Jullier e Leveratto 2010), também no âmbito dos festivais (De Valck 2005, 2010). As novas práticas cinéfilas, heterogéneas, estão, aliás, como aponta Christel Taillibert, intimamente relacionadas com a crescente especialização dos festivais $(2009,46)$.

No que diz respeito às aproximações metodológicas, Loist tem refletido sobre as especificidades de que se reveste o estudo dos festivais, que tende a aliar metodologias qualitativas e quantitativas, embora as primeiras sejam predominantes (2016). Por um lado, as investigações históricas dependem, naturalmente, de arquivos, os quais podem oferecer inestimáveis recursos (Berbain e Bokova 2013; Barnes 2020), mas também apresentam inúmeros problemas e limitações (Zielinski 2016; Barnes 2020; Peirano 2020a). Devem, por isso, ser combinados e contrastados com a história oral (Peirano 2020a). No caso da pesquisa sobre práticas contemporâneas, a etnografia tem sido a ferramenta privilegiada, permitindo superar algumas limitações que surgem ao aplicar outras metodologias das ciências sociais (Leão 2006; Peirano 2020a). A antropologia oferece um quadro conceptual que nos permite analisar a 
operacionalidade e os discursos não oficiais dos festivais, aplicando conceitos como "facto social total", etnografia multissituada, papéis e normas de comportamento, etnografia como forma de escrita, ou as abordagens etic/ emic (insider/ outsider) (Vallejo 2017a). Efetivamente, o posicionamento do pesquisador e as limitações de acesso aos dados internos dos festivais são fundamentais para compreender as possibilidades e obstáculos metodológicos ao estudo dos festivais (Loist 2020). Esta questão é de especial relevância dada a estreita colaboração entre pesquisadores/as e festivais, já que vários elementos de universidades foram fundadores, diretores, programadores ou arquivistas destes eventos, bem como membros do júri ou participantes de mesas redondas e publicações. Além disso, a chamada pesquisa baseada na prática (com projetos de doutoramento colaborativos entre universidades e festivais) oferece mais um passo em direção à institucionalização desses modos de colaboração, como podemos ver nos trabalhos desenvolvidos, na Escócia, por Lesley-Ann Dickson, com o Festival de Cinema de Glasgow (2014, 2017a), e Alexandra Colta, com o Document Human Rights Film Festival (2019a).

Mais recentemente, o espaço social virtual tem vindo a ganhar especial relevância, e algumas pesquisas têm vindo a dar enfoque aos fóruns da internet e redes sociais (como o Facebook ou o Twitter), encarando-os como espaços sociais que têm contribuído para a criação de iniciativas profissionais (Battaglia 2020), mas também de discursos em torno dos festivais (Dougados et al. 2014; Burgess 2020). Esta orientação é, sem dúvida, particularmente interessante no panorama atual, uma vez que as medidas de confinamento e a limitação da mobilidade implementadas em resposta à pandemia da COVID-19 levaram a que grande parte da interação social que ocorre nos festivais se transpusesse para as redes sociais. Além do mais, a evolução das Humanidades Digitais tem vindo a disponibilizar novas ferramentas, não só para a realização de análise de conteúdo online, mas também para a apresentação visual dos resultados, através de representações gráficas que permitem dar forma às hierarquias e relações dentro do circuito. Podemos observá-lo no projeto de investigação sobre circulação de filmes dirigido por Skadi Loist (Loist e Samoilova 2020) ou no gráfico de redes sociais de cineastas da América Central, desenvolvido por Jasper Vanhaelemeesch $(2020,127)$.

Nos estudos dedicados a festivais específicos, verifica-se uma influência especial da antropologia. Já na década de 1950 surgem os primeiros textos que refletem sobre a vertente mais social do Festival de Cannes, ora 
associando as suas formas de funcionamento a uma ordem religiosa (Bazin 1955), ora destacando a crescente importância da dimensão social (e do papel da presença de 'estrelas'), em detrimento dos filmes (Morin 1955). Quase meio século depois, Daniel Dayan (2000) presenteava-nos com uma leitura antropológica das categorias de participantes e dos seus discursos, no festival de Sundance, naquele que foi um contributo determinante no olhar sobre a componente social dos festivais e na identificação dos vários agentes sociais que neles participam. As pessoas que trabalham em festivais (na sua organização, programação ou comunicação) têm sido objeto de vários estudos. Estes incluem textos e reflexões sobre as condições de trabalho do setor (Taillibert 2009: 241-249; Loist 2011; Dickson 2018; Colta 2019b), bem como estudos dedicados à criação das elites culturais de críticos, diretores e programadores, cujas relações e práticas sociais foram fundamentais para definir o rumo dos festivais de maior repercussão internacional (Jungen 2014, 83-97; 2020), servindo de tradutores culturais e "guardiães" (gatekeepers) que decidem a entrada de cinematografias de todo o mundo no circuito dos festivais, como Dovey aponta para o caso africano (2015). Por outro lado, os discursos criados por e em torno dos festivais foram objeto de diversas análises etnográficas, como o trabalho de Toby Lee sobre o contexto grego no âmbito da publicação do livro

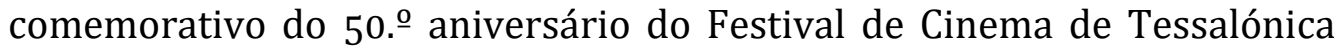
(2005), ou o estudo de Sarah Mitchell sobre as estratégias de gestão da informação implementadas pelo Toronto International Film Festival (2017). Outras pesquisas ainda colocam o enfoque nos profissionais que frequentam os festivais de cinema. Como veremos nos estudos citados de seguida, tais profissionais podem ser políticos e representantes institucionais, intelectuais e personalidades do universo da cultura, cineastas, e, mais recentemente, produtores/as, agentes de vendas e outros trabalhadores/as da indústria audiovisual, o que evidencia o papel do festival como espaço de negociação cultural, mas também de negociação política e económica.

Os contextos sociopolíticos têm sido, por sua vez, objeto de várias pesquisas históricas sobre festivais de cinema, onde predominam os trabalhos sobre a Guerra Fria e as práticas diplomáticas, de espionagem ou de controle policial daquele período (Kötzing e Moine 2017; Pisu 2013; Fehrenbach 2019; Bláhová 2019; Razlogova 2015 e 2020; García Rivas 2020). Há a assinalar as pesquisas centradas nos festivais independentes que nasceram com os movimentos sociais difundidos nos anos 70 do século passado. Estes trabalhos visaram reconstruir o papel de determinados festivais como espaços sociais de negociação de ideologias. Para tal, exploraram a 
importância dos processos de colaboração, discussão e troca de informações desenvolvidos nestes contextos. Foi o caso das obras de Mariano Mestman sobre o Terceiro Cinema (2002, 2016a, 2016b) ou de Antoine Damiens sobre o cinema feminista (2020b). Neste contexto, os estudos de públicos que exploraram o ativismo vinculado a festivais de direitos humanos ou a outros eventos sociais são particularmente interessantes (Iordanova e Torchin 2012; Roy 2014; Tascón 2015; Tascón e Wils 2017).

Os estudos dedicados às práticas contemporâneas têm analisado, a partir de uma perspetiva etnográfica, as redes de cineastas e profissionais que circulam em festivais internacionais, seja em relação ao cinema nacional (ver Peirano 2017b, 2018, 2019 e 2020b, para o caso chileno) ou regional (ver Vanhaelemeesch 2020, para a América Central), ou, ainda, em relação a géneros cinematográficos, como o cinema documental (Vallejo 2015, 2017b). Há também as investigações centradas nas redes nacionais de profissionais e nas distintas formas de ativismo cultural em torno de festivais locais em países como a China (Lichaa 2017; Leung Cheung 2020) ou a Índia (Battaglia 2020). No entanto, as dinâmicas internas de comportamento dos elementos do júri, não obstante o seu elevado interesse para se compreender a influência das relações sociais nos processos de criação de valor simbólico em festivais, têm originado uma quantidade reduzida de pesquisas. Entre estas, encontramos trabalhos, esporádicos, dedicados a festivais especializados como os de cinema documental (De Valck e Soeteman 2010) ou cinema etnográfico (Piault 2017).

Por fim, os públicos. Os públicos têm sido o objeto de diversas pesquisas, que têm analisado as distintas formas destes se relacionarem e participarem num festival. Aqui, faz sentido destacar o livro editado pelo sociólogo francês Emmanuel Ethis (2001). Inovadora à época da sua publicação, a obra oferece contributos de vários autores que, a partir de abordagens socioantropológicas, tecem um amplo panorama dos rituais, papéis, formas de comportamento ou modelos de participação no festival de Cannes, combinando reflexões mais conceptuais com dados estatísticos, recolhidos através de inquéritos por questionário aplicados entre 1999 e 2000. Ethis, que estudou os públicos do festival de teatro de Avignon e do festival de cinema de Cannes, publicou, recentemente, um novo volume coletivo, que descreve diferentes perfis de tipos de público de ambos os festivais, recorrendo ao que o autor denomina de "sociogramas" (Ethis 2011). De resto, tal como referíramos anteriormente, nos últimos anos as 
redes sociais passaram a ocupar um lugar central na análise dos públicos (Ethis e Poli 2014; Stevens 2018).

Dentre os estudos mais recentes dedicados a festivais de menor impacto, destacam-se algumas teses de doutoramento: é o caso da pesquisa sobre o público do Festival de Cinema de Glasgow, realizado entre 2011 e 2013, período em que o festival aumentou a sua audiência de 6.000 para 39.000 visitantes (Dickson 2014, 2015, 2017b); do estudo de Rosana Vivar Navas sobre a Semana de Cinema Fantástico e de Terror de San Sebastián (2016, 2017); ou de Tânia Leão, que compara as modalidades de participação dos públicos do IndieLisboa, festival de cinema 'independente', e do Curtas Vila do Conde, especializado em curtas-metragens (2019). Nestes estudos, encontramos uma combinação de métodos e técnicas, que passam pelos grupos focais, observação participante e entrevistas. No caso da pesquisa de Leão, a abordagem metodológica é mista, aliando métodos e técnicas qualitativos (onde se inclui a etnografia) e quantitativos. Aliás, são precisamente os festivais especializados aqueles em que o sentido de comunidade e os rituais (re)criados pelo público são de especial interesse. Estes têm sido objeto de alguns estudos, sendo de salientar, pela sua absoluta singularidade, os festivais de filmes de cinema fantástico e de terror (Stringer 2008; Leão 2006, 2007/2008, 2008), alguns tendo sublinhado as relações de género, por exemplo, no que toca às formas de negociação da representação feminina (Vivar 2019) ou da masculinidade (Frohlick 2015). Finalmente, apesar de não se tratarem de estudos académicos stricto sensu, importa mencionar os relatórios de estudos de públicos encomendados por festivais específicos, como é o caso do festival de documentário Hot Docs, de Toronto, onde não apenas se analisaram os públicos do festival, mas também aqueles que "não assistem a documentários", para identificar possíveis estratégias de expansão de públicos (De Rosa e Burgess 2014).

A dimensão mais social dos festivais - como espaços de encontro, aprendizagem, negociação ou trabalho -, bem como as formas como esses espaços e processos se entrelaçam com os objetos culturais (os filmes) e contribuem para a sua legitimação são elementos que perpassam todos os textos da atual publicação. Os seis artigos incluídos neste dossier fornecem novas perspectivas e acrescentam estudos de caso ao prolífico campo de estudo dos festivais de cinema, e serão apresentados a seguir. 


\section{Conteúdos}

Os artigos que constam da presente publicação revelam a diversidade de abordagens possibilitada pelo estudo dos festivais. Neles, entrecruzam-se análises com diferentes enfoques metodológicos, que combinam a perspetiva histórica, a aproximação etnográfica, a análise dos discursos dos media, das estratégias curatoriais ou das políticas governamentais. Além disso, a heterogeneidade geográfica dos casos apresentados - Chile, Brasil, Senegal, Galiza (Espanha) e Portugal - dá-nos uma visão mais global, que permite comparar processos que, embora possam ter características semelhantes, revelam as suas peculiaridades locais e uma forte relação com a criação de espaços culturais vinculados a diferentes países, culturas, línguas, géneros cinematográficos ou comunidades cinéfilas. 0 primeiro artigo, assinado por Rafael Zanatto (Universidade de São Paulo), propõe uma análise histórica do I Festival Internacional de Cinema do Brasil (1954). A avaliação que o autor faz dos mecanismos de legitimação do festival "falido" (que não sobreviveu à primeira edição) revela o confronto entre a posição institucional, inclinada para uma dimensão mais comercial do cinema, e os agentes culturais da época, com aspirações de presentear o público local com uma iniciativa de cariz propriamente cinéfilo. 0 texto seguinte, de María Paz Peirano (Universidade do Chile), aprofunda a dimensão social dos festivais de cinema do seu país. A autora analisa momentos-chave para a formação de grupos cinéfilos, isto é, de grupos que constituem o "núcleo duro" dos profissionais de cinema: cineastas, programadores, críticos e agentes da indústria. Com foco no Festival Internacional de Cinema de Viña del Mar, na década de 1990, e no Festival Internacional de Cinema de Valdivia, na década de 2000, o artigo revela o papel desses eventos como pontos de encontro e espaços de consenso sobre o cânone do cinema chileno de diferentes períodos. Já o artigo assinado por Tânia Leão (Instituto de Sociologia da Universidade do Porto) procura esboçar um panorama global dos festivais de cinema em Portugal, do seu nascimento e evolução. Através de um itinerário histórico, a autora oferece um olhar sobre as múltiplas facetas destes acontecimentos, aprofundandose a análise nas particularidades do contexto político e cultural do país e na relação deste contexto com as diversas alterações legislativas, e com a indústria audiovisual local. 0 texto pretende, ainda, sinalizar lacunas nos estudos sobre festivais de cinema em Portugal e incentivar novas pesquisas. Seguidamente, o texto de Fernando Redondo Neira (Universidade de Santiago de Compostela) centra-se no papel que os/as programadores/as e a crítica têm tido na articulação do movimento cinematográfico denominado "Novo Cinema Galego". Partindo da análise do trabalho de 
críticos, programadores e cineastas, o autor mostra como certas cinematografias periféricas (e, mais especificamente, aquelas que são feitas em línguas minoritárias, como é o caso do cinema galego) podem viajar globalmente, devido ao papel determinante desempenhado pelos circuitos de festivais locais e internacionais (e suas conexões). A historiadora Juliana Mylaert Mager (Universidade Federal Fluminense) traz-nos um artigo dedicado ao festival de cinema documental É Tudo Verdade. Através da análise das estratégias curatoriais do festival, a autora analisa o cânone do documentário brasileiro proposto pelo festival nas suas retrospectivas, publicações e atividades paralelas, comparando-o com cânones alternativos propostos por outros festivais do país, como o Fórumdoc, o Recine ou o Cachoeiradoc. 0 último artigo, da autoria da antropóloga Estrella Sendra (SOAS, University of London/ University of Southampton), analisa a trajetória do Banlieue Films Festival em Dakar (Senegal), criado em 2013. Recorrendo a uma análise do papel do seu fundador, Abdel Aziz Boye, a autora estuda os processos de criação de um espaço cultural e de formação que tem sido determinante para a evolução da carreira de novos/as cineastas no país.

Os trabalhos aqui propostos contribuem para uma melhor compreensão dos contextos socioculturais em que se desenvolveram as várias culturas cinematográficas, centrando-se nos principais agentes e profissionais do cinema (crítica, curadoria, organização, legislação). Embora respondam a algumas das áreas de interesse propostas pelo dossier, fica claro que outras ainda precisam de ser exploradas. Em particular, a dimensão dos públicos, dos seus comportamentos ou do efeito que os festivais podem ter nas suas atitudes - não só no domínio do consumo cultural e da cinefilia, mas também no do ativismo político ou da construção de identidades específicas. Da mesma forma, uma abertura do estudo dos festivais a novos espaços geopolíticos apenas enriquecerá o âmbito de análise que aqui apresentamos. Desejamos que este seja mais um passo para alargar os horizontes dos estudos de festivais de cinema, especialmente nos âmbitos lusófono e de língua espanhola.

\section{Referências}

Barnes, Heather L. 2020. "The Data-driven Festival: Recordkeeping and Archival Practices." Em Documentary Film Festivals Vol. 1. Methods, History, Politics, editado por Aida Vallejo, e Ezra Winton, 53-59. Cham: Palgrave Macmillan. 
Battaglia, Giulia. 2020. "The Development of Documentary Film Festivals in India: A Small-Media Phenomenon." Em Documentary Film Festivals Vol 1: Methods, History, Politics, editado por Aida Vallejo, e Ezra Winton, 221-239. Cham: Palgrave MacMillan.

Bazin, André. 1955. "Du festival considéré comme un ordre," Les Cahiers du cinéma, 54-56.

Berbain, Iris e Bokova, Lenka. 2013. “Quelles sources pour l'histoire des festivals?: Les collections du départment des Arts du spectacle de la BnF; l'histoire du festival d'Avignon dans les collections de la maison Jean Vilar". Em Une histoire des festivals: XXe-XXIe siècle, editado por Anaïs Fléchet, Pascale Gœtschel, Patricia Hidiroglou, Sophie Jacotot, Caroline Moine, e Julie Verlaine, 303-315. Paris: Publications de la Sorbonne.

Biltereyst, Daniel, Maltby, Richard e Meers, Philippe. 2019. The Routledge Companion to New Cinema History. Londres: Routledge.

Bláhová, Jindřiška. 2019. "Political significance of a Butcher in Love: the 1956 Karlovy Vary international Film Festival, Marty (1955) and the Restoration of Contact between Hollywood and Czechoslovakia during the Cold War." Studies in European Cinema 17 (2): 97-112.

Burgess, Diane. 2020. "Capturing film festival buzz: The methodological dilemma of measuring symbolic value." NECSUS. European Journal of Media Studies 9 (2): 225-247.

Campos, Minerva. 2012. "El circuito de financiación de los cines latinoamericanos." Cinémas d'Amérique Latine 20: 172-180.

. 2020. "Tensiones en el circuito cinematográfico internacional: modelo para el estudio de los festivales latinoamericanos." Comunicación y Medios 42: 72-84.

Colta, Alexandra. 2019a. Politics and Programming Practices at Human Rights Film Festivals: A Study of Document Human Rights Film Festival. Tese de doutoramento. University of Glasgow. . 2019b. "Creative and Emotional Labour: Programming human rights film festivals as practice-led ethnography." Alphaville: Journal of Film and Screen Media 17: 128-145.

Damiens, Antoine. 2020a. LGBTQ Film Festivals: Curating Queerness. Amsterdam: Amsterdam University Press. 
2020b. "Film Festivals of the 1970s and the Subject of Feminist Film Studies: Collaborations and Regimes of Knowledge Production." Journal of Film and Video 72 (1-2): 21-32.

Dayan, Daniel. 2000. "Looking for Sundance: The Social Construction of a Film Festival." Em Moving Images, Culture and the Mind, editado por Ib Bondebjerg, 43-52. Luton: University of Luton Press.

De Rosa, Maria e Burgess, Marilyn. 2014. Learning from Documentary Audiences: A Market Research Study. Toronto: Hot Docs Canadian International Documentary Film Festival. https://goo.gl/dVndHQ. Acedido em Dezembro de 2020.

De Valck, Marijke e Soeteman, Mimi. 2010. '“And the Winner is...' What Happens Behind the Scenes of Film Festival Competitions." International Journal of Cultural Studies 13 (3): 290-307.

De Valck, Marijke, Kredell, Brendal e Loist, Skadi. 2016. Film Festivals: History, Theory, Method, Practice. Londres, Nova Iorque: Routledge.

De Valck, Marijke. 2005. "Drowning in Popcorn at the International Film Festival Rotterdam? The Festival as a Multiplex of Cinephilia." Em Cinephilia: Movies, Love and Memory, editado por Marijke de Valck e Malte Hagener, 97-109. Amsterdam: Amsterdam University Press. . 2007. Film Festivals. From European Geopolitics to Global Cinephilia. Amsterdam: Amsterdam University Press.

. 2010. "Reflections on the Recent Cinephilia Debates." Cinema Journal 49 (2): 132-139.

. 2012. "Finding Audiences for Films: Programming in Historical Perspective". Em Coming Soon to a Festival Near You: Programming Film Festivals, editado por Jeffrey Ruoff, 25-40. St Andrews: St Andrews Film Studies.

. 2020. "Vulnerabilities and Resiliency in the Festival Ecosystem: Notes on Approaching Film Festivals in Pandemic Times". Em Pandemic Media: Preliminary Notes Towards an Inventory, editado por Phillipp Dominik Keidl, Laliv Melamed, Vinzenz Hediger, e Antonio Somaini. Lüneburg: Meson Press. https://pandemicmedia.meson.press/chapters/spacescale/vulnerabilities-and-resiliency-in-the-festival-ecosystemnotes-on-approaching-film-festivals-in-pandemic-times/ . Acedido em Janeiro de 2021. 
Dickson, Lesley-Ann. 2014. Film Festival and Cinema Audiences: A Study of Exhibition Practice and Audience Reception at Glasgow Film Festival. Tese de doutoramento, University of Glasgow. . 2015. “'Ah! Other Bodies!': Embodied spaces, pleasures and practices at Glasgow Film Festival”. Participations: Journal of Audience and Reception Studies 12 (1): 703-724.

. 2017a. "Insider/ outsider positions at Glasgow Film Festival: Challenges, issues and opportunities in industry-partnered ethnographic research". Em Film Festivals and Anthropology, editado por Aida Vallejo e María Paz Peirano, 261-276. Newcastle upon Tyne: Cambridge Scholars Publishing.

. 2017b. “'Beyond Film' Experience: Festivalizing Practices and Shifting Spectatorship at Glasgow Film Festival". Em Live Cinema: Cultures, Economies, Aesthetics, editado por Sarah Atkinson, e Helen W. Kennedy, 83-100. London: Bloomsbury Academic.

. 2018. "Episodic Volunteer Management at Festivals: The case of Valletta Film Festival, Valetta, Malta." Em Managing Organisational Success in the Arts, editado por David Stevenson, 147-170. Oxford: Routledge.

Dougados, Myriam, Fabiani, Jean-Louis, Gaillard, Julien e Portilla, Yonathan. 2014. "Les usages de Twitter au festival de Cannes. Pratiques numériques et construction des opinions esthétiques." Culture \& Musées 24: 89-106.

Dovey, Lindiwe. 2015. Curating Africa in the Age of Film Festivals: Film Festivals, Time, Resistance. Londres, Nueva York: Palgrave Macmillan.

Ethis, Emmanuel e Poli, Marie-Sylvie. 2014. "Hopper 2013/ Cannes 2013 au prisme des écritures numériques." Culture \& Musées 24: 63-88.

Ethis, Emmanuel. 2001. Aux marches du palais: Le festival de Cannes sous le regard des sciences sociales. Paris: La Documentation Française. 2011. La Petite fabrique du spectateur. Être et devenir festivalier à Cannes et à Avignon. Avignon: Éditions universitaires d'Avignon.

Falicov, Tamara L. 2010. "Migrating from South to North: The Role of Film Festivals in Funding and Shaping Global South Film and Video." Em Locating Migrating Media, editado por Greg Elmer, Charles H. Davis, Janine Marchessault, e John McCollough, 3-22. Lanham: Lexington Books. 
2017. "Film Funding Opportunities for Latin American Filmmakers: A Case for Further North-South Collaboration in Training and Film Festival Initiatives." Em A Companion to Latin American Cinema, editado por María M. Delgado, Stephen M. Hart, e Randan Johnson, 85-98. Chichester, W. Sussex: Wiley Blackwell.

Fehrenbach, Heide. 2019. "The Berlin International Film Festival: Between cold war politics and postwar reorientation." Studies in European Cinema 17 (2): 81-96.

Frohlick, Susan. 2005. “That Playfulness of white masculinity': Mediating masculinities and adventure at mountain film festivals." Tourist Studies 5: 175-93.

García Rivas, Carlos Daniel. 2020. "Estrategias de vigilancia policial en el Festival Internacional de Cine de Mar del Plata entre 1959 y 1960." Comunicación y Medios 42: 85-95.

Harbord, Janet. 2002. Film Cultures. London: Sage.

Iordanova, Dina e Torchin, Leshu. 2012. Film Festival Yearbook 4: Film Festivals and Activism. St Andrews: St Andrews Film Studies.

Iordanova, Dina e Cheung, Ruby. 2011. Film Festival Yearbook 3: Film Festivals and East Asia. St. Andrews: St. Andrews Film Studies.

Iordanova, Dina. 2013. The Film Festival Reader. St Andrews: St Andrews Film Studies.

. 2017. "Yingying, Zhenzhen, and Fenfen? China at the Festivals." Em Chinese Film Festivals: Sites of Translation, editado por Chris Berry, e Luke Robinson, 217-235. Nueva York: Palgrave Macmillan.

Jullier, Laurent e Leveratto, Jean-Marc. 2010. Cinéphiles et cinéphilies. Une histoire de la qualité cinématographique. Paris: Armand Colin.

Jungen, Christian. 2014. Hollywood in Cannes. The history of a love-hate relationship. Amsterdam: Amsterdam University Press.

. 2020. "Forging a Cultural Elite: Nyon and the Age of Festival Programmers." Em Documentary Film Festivals Vol 1: Methods, History, Politics, editado por Aida Vallejo e Ezra Winton, 123-141. Cham: Palgrave MacMillan.

Jurado-Martín, Montserrat. 2003. Los festivales de cine en España: incidencia en los nuevos realizadores y análisis del tratamiento que reciben en los medios de comunicación. Tese de doutoramento. Universidad Complutense de Madrid. 
Kötzing, Andreas e Moine, Caroline. 2017. Cultural Transfer and Political Conflicts. Film Festivals in the Cold War. Göttingen: V\&R unipress $\mathrm{GmbH}$.

Leal, Antonio e Mattos, Tetê. 2011. Painel Setorial dos Festivais Audiovisuais, Indicadores 2007, 2008, 2009. Rio de Janeiro: IBEFEST/Fórum dos Festivais/ASSOCIAÇÃo Cultural Kinoforum.

Lee, Toby. 2016. "Being There, Taking Place: Ethnography at the Film Festival." Em Film Festivals: History, Theory, Method, Practice, editado por Marijke de Valck, Brendan Kredell, e Skadi Loist, 122-137. Londres e Nova York: Routledge.

Leung Cheung, Tit. 2020. "The Film Festival of Independent and Underground: The Case of DOChina." Em Documentary Film Festivals Vol 1: Methods, History, Politics, editado por Aida Vallejo e Ezra Winton, 175-191. Cham: Palgrave MacMillan.

Leão, Tânia. 2006. O Fantástico e os Seus Públicos: O Festival Internacional de Cinema do Porto Como Espaço Multivocal. Dissertação de Mestrado em Comunicação, Cultura e Tecnologias da Informação. Lisboa: ISCTE-IUL.

2007. “O(s) Público(s) do Fantasporto: Perfis-tipo de modalidades de apropriação ritualista do Festival Internacional de Cinema do Porto". Trajectos 11: 31-44.

. 2007/2008. “O Fantástico em Análise: Algumas notas sobre o estudo compreensivo da 23. a edição do Fantasporto". Sociologia: Revista da Faculdade de Letras da Universidade do Porto 17-18: 81-109.

. 2019. Públicos de Festivais de Cinema em Portugal: Um estudo comparado. Tese de doutoramento em Sociologia: Cidades e Culturas Urbanas. Coimbra: Faculdade de Economia da Universidade de Coimbra.

Lichaa, Flora. 2017. "A Community at the Margins: An Ethnography of Chinese Independent Film Festivals." Em Film Festivals and Anthropology, editado por Aida Vallejo, e María Paz Peirano, 293304. Newcastle upon Tyne, England: Cambridge Scholars Publishing.

Loist, Skadi e Samoilova, Zhenya. 2020. "First Results from Our Survey of Filmmakers on How Their Films Traveled Through Festivals." Film Circulation, 92020. www.filmcirculation.net/2020/01/09/first-results-from-oursurvey-of-filmmakers-on-how-their-films-traveled-throughfestivals/ . Acedido em Dezembro de 2020. 
Loist, Skadi e Zielinski, Ger. 2012. "On the Development of Queer Film Festivals and Their Media Activism." Em Film Festival Yearbook 4: Film Festivals and Activism, editado por Dina Iordanova, e Leshu Torchin, 49-62. St Andrews: St Andrews Film Studies.

Loist, Skadi. 2011. "Precarious Cultural Work: About the Organization of (Queer) Film Festivals." Screen 52:2: 268-273.

. 2013. "The Queer Film Festival Phenomenon in a Global Historical Perspective (the 1970s-2000s)." Em Une histoire des festivals: XXeXXIe siècle, editado por Anaïs Fléchet, Pascale Gœtschel, Patricia Hidiroglou, Sophie Jacotot, Caroline Moine, e Julie Verlaine, 109-121. Paris: Publications de la Sorbonne.

. 2016. "The film festival circuit: networks, hierarchies, and circulation." Em Film Festivals: History, Theory, Method, Practice, editado por Marijke de Valck, Brendan Kredell, e Skadi Loist, 49- 64. Londres: Routledge.

- 2020. "Film Festival Research Workshops: Debates on Methodology." Em Documentary Film Festivals Vol 1: Methods, History, Politics, editado por Aida Vallejo, e Ezra Winton, 41-52. Cham: Palgrave MacMillan.

Mattos, Tetê e Leal, Antonio. 2009. "Festivais audiovisuais brasileiros: um diagnóstico do setor." Em Políticas Culturais: Reflexões e ações, editado por Lia Calabre, 201-223. São Paulo/Rio de Janeiro: Itaú Cultural/Fundação Casa Rui de Barbosa.

Mestman, Mariano. 2002. "From Algiers to Buenos Aires: The Third World Cinema Committee (1973-74)." New Cinemas: Journal of Contemporary Film, 1 (1): 40-53

Mestman, Mariano. 2016a. "Argel, Buenos Aires, Montreal: El comité de cine del Tercer Mundo (revisión actualizada)." Secuencias. Revista de Historia del Cine, 43-44: 73-93.

Mestman, Mariano. 2016b. Las Rupturas del 68 en el cine de América Latina. Buenos Aires: Ediciones Akal.

Mitchell, SED. 2017. "Programmer as Festival Spokesperson: Information Management Strategies at the Toronto International Film Festival." Em Film Festivals and Anthropology, editado por Aida Vallejo, e María Paz Peirano, 305-321. Newcastle upon Tyne: Cambridge Scholars Publishing. 
Morin, Edgar. 1955. "Notes pour une sociologie du festival de Cannes." Temps modernes 10 (114-115): 2273-2284.

Peirano, Maria Paz e González, Sebastián. 2018. Los Festivales de Cine en Chile (1963/1967-2017). Informe Festivales de Cine en Chile: ventanas de exhibición y difusión del cine chileno.

Peirano, María Paz. 2017a. "Mapping Ethnographic Film Festivals: A World Overview." Em Film Festivals and Anthropology, editado por Aida Vallejo, e María Paz Peirano, 21-36. Newcastle upon Tyne: Cambridge Scholars Publishing.

. 2017b. "Film mobilities and Circulation practices in the construction of recent Chilean Cinema." Em Envisioning Networked Urban Mobilities. Art, Performances, Impacts, editado por Aslak Aamot Kjærulff, Sven Kesselring, Peter Peters, e Kevin Hannam, 35-47. Nova York: Routledge.

. 2019. "Filmmakers as "citizens of the world": Cosmopolitanism and global identities of the Chilean upper-middle class 1." Em Citizenship in the Latin American Upper and Middle Classes, editado por Fiorella Montero-Diaz, e Franka Winter, 46-61. Nova York: Routledge.

. 2018. "Festivales de cine y procesos de internacionalización del cine chileno reciente." Cuadernos.info 43: 57-69.

2020a. "Mapping histories and archiving ephemeral landscapes: strategies and challenges for researching small film festivals." Studies in European Cinema 17 (2): 170-184.

. 2020b. "Connecting and sharing experiences: Chilean documentary film professionals at the film festival circuit." Em Documentary Film Festivals Vol 2: Changes, Challenges, Professional Perspectives, editado por Aida Vallejo, e Ezra Winton, 55-72. Cham: Palgrave MacMillan.

Piault, Colette. 2017. "Festivals, Conferences, Seminars and Networks in Visual Anthropology in Europe." Em Film Festivals and Anthropology, editado por Aida Vallejo e María Paz Peirano, 39-72. Newcastle upon Tyne: Cambridge Scholars Publishing.

Pisu, Stefano. 2013. Stalin a Venezia. L'Urss alla Mostra del cinema fra diplomacia culturale e scontro ideologico (1932-1953). Catanzaro: Rubbettino Editore.

Razlogova, Elena. 2015. "The Politics of Translation at Soviet Film Festivals during the Cold War." SubStance 44 (2): 66-87. 
2020. "World cinema at Soviet festivals: cultural diplomacy and personal ties." Studies in European Cinema 17 (2): 140-154.

Rhyne, Ragan. 2018. "Queer Capital, Queer Culture: Gay and Lesbian Film Festivals in the 1990s." Em International Film Festivals: Contemporary Cultures and History Beyond Venice and Cannes, editado por Tricia Jenkins, 153-178. London: I.B. Tauris.

Richards, Stuart James. 2016. The Queer Film Festival: Popcorn and Politics. Nueva York: Palgrave Macmillan.

Roy, Carole. 2014. Documentary Film Festivals. Transformative Learning, Community Building \& Solidarity. Rotterdam: Sense Publishers.

Stevens, Kirsten 2018. "You had to be there: Film Festival 'Liveness' and the Digitally Connected Audience." Em International Film Festivals. Contemporary Cultures and History Beyond Venice and Cannes, editado por Tricia Jenkins, 11-31. Londres: I.B. Tauris.

Taillibert, Christel. 2009. Tribulations Festivalières: Les festivals de cinéma et audio-visuel en France. Paris: L'Harmattan.

Tascón, Sonia M. 2015. Human Rights Film Festivals: Activism in Context. London and New York: Palgrave Macmillan.

Tascón, Sonia M. e Wils, Tyson. 2017. Activist Film Festivals: Towards a Political Subject. Chicago: University of Chicago Press.

Vallejo, Aida. 2014. "Festivales cinematográficos: en el punto de mira de la historiografía fílmica." Secuencias. Revista de Historia del cine 39: 1342.

2015. "Documentary Filmmakers on the Circuit. A Festival Career from Czech Dream to Czech Peace." Em Post-1990 Documentary. Reconfiguring Independence, editado por Camille Deprez, e Judith Pernin, 171-187. Edinburgh: Edinburgh University Press.

2017a. "Ethnographies of Film Festivals: Reflections on Methodology." Em Film Festivals and Anthropology, editado por Aida Vallejo, e María Paz Peirano, 251-260. Newcastle upon Tyne: Cambridge Scholars Publishing.

. 2017b. "“'Travelling the Circuit: A Multi-sited Ethnography of Documentary Film Festivals in Europe." Em Film Festivals and Anthropology, editado por Aida Vallejo, e María Paz Peirano, 277-292. Newcastle upon Tyne: Cambridge Scholars Publishing.

. 2020a. "Introduction to Part II, Vol. 1: Mapping the History of Documentary Film Festivals." Em Documentary Film Festivals Vol 1:

anikiDossier temático: Festivais de Cinema | Special section: Film Festivals 
Methods, History, Politics, editado por Aida Vallejo, e Ezra Winton, 6375. Cham: Palgrave MacMillan.

2020b. "Rethinking the Canon: the Role of Film Festivals in Shaping Film History." Studies in European Cinema 17 (2): 155-169.

Vanhaelemeesch, Jasper. 2020. "The seeds multiply: the AcampaDOC International Documentary Film Festival." Comunicación y Medios 42: $120-133$.

Vivar, Rosana. 2016. Los festivales de cine en la era de los new media: una perspectiva lúdica sobre la fiesta del cine y sus públicos. Tese de doutoramento. Universidad de Granada.

- 2017. "Never Seen a Shot Like that Before! Playfulness and Participatory Audiences in San Sebastian Horror and Fantasy Film Festival". Em Live Cinema: Cultures, Economies, Aesthetics, editado por Sarah Atkinson, e Helen W. Kennedy, 117-132. New York: Bloomsbury Academic.

. 2019. "You're Sick if that Turns you on!': Female Participation and Gender Identities at San Sebastian Horror and Fantasy Film Festival." Em Gender and Contemporary Horror in Comics, Games and Transmedia, editado por Robert Shail, Samantha Holland, e Steven Gerrard, 151-165. Bingley: Emerald Publishing Limited.

Wong, Cindy Hing-Yuk. 2011. Film Festivals. Culture, People, and Power on the Global Screen. London: Rutgers University Press.

Zielinski, Ger. 2016. "On Studying Film Festival Ephemera: The Case of Queer Film Festivals and Archives of Feelings." Em Film Festivals: History, Theory, Method, Practice, editado por Marijke de Valck, Brendan Kredell, e Skadi Loist, 138-158. Londres e Nova York: Routledge. 